\section{Left to right shunts with pulmonary vascular disease, still an enigma}

\author{
R Krishna Kumar
}

Pulmonary vascular obstructive disease (PVOD) is a feared complication of congenital heart disease (CHD) associated with increased pulmonary blood flow. It presumably develops as a protective response to prevent pulmonary overcirculation in these patients. However, with time the changes become irreversible and, in advanced stages, correction of the defect is rendered futile and potentially hazardous. In most low-income and middle-income countries, comprehensive paediatric heart care has only recently become available. Because of serious paucity of resources, health system dysfunctions and limited awareness, only a small minority of infants and children with CHD receive timely correction. ${ }^{1}$ In spite of substantial early attrition there is still a large population of older patients with uncorrected CHD. A significant proportion of these are patients with uncorrected large left to right shunts and increased pulmonary resistance with PVOD. ${ }^{2}$ Because the response of the pulmonary vasculature to high pulmonary blood flow is not uniform and does not occur in a predictable fashion, a spectrum of possibilities is often seen. Thus it is possible to encounter an adult with a large ventricular septal defect or patent arterial duct who may still be operable and would benefit from closure of the defect. It is also possible to see an infant with ventricular septal defect who may not be operable because of advanced PVOD. Decision-making is typically straightforward at the extreme ends of the spectrum. Patients who have an obvious resting pulmonary overcirculation that is often identifiable through clinical examination, chest X-ray and echocardiography can be offered correction with the expectation that they would be left with little or no pulmonary hypertension. At the other end are patients with frank Eisenmenger syndrome and significant resting desaturation where it is clinically obvious that closure would be hazardous. There are a significant number of patients who fall in

Correspondence to Dr R Krishna Kumar, Clinical Professor and Head, Pediatric Cardiology, Amrita Institute of Medical Sciences and Research Centre, Cochin, 682041, Kerala, India; rkrishnakumar@aims. amrita.edu, kumar_rk@yahoo.com the grey zone between operable and inoperable status. $^{2} 3$ These are patients with borderline data where there is often a temptation to offer correction with the 'hope of cure', particularly with improving overall surgical outcomes and after availability of selective pulmonary vasodilators. Most surgeons choose to leave behind a small fenestration in the closure patch to allow a safe and uncomplicated recovery in the early postoperative period. This fenestration serves as a pop-off in the event of a substantial rise in pulmonary artery pressures and, if needed, this fenestration can be potentially closed in the catheterisation laboratory by an occlusive device at a later date.

The review by Talwar et al ${ }^{4}$ seeks to describe the problem of CHD with PVOD and outline surgical options for patients with borderline operability. In particular, they discuss at length the use of the unidirectional-valved patch (UVP) for closure of defects. These patches are designed to serve as a pop-off whenever pulmonary arterial pressures rise to unacceptable levels without allowing any left to right shunt whatsoever. The premise behind using a UVP is quite sound. The 'all India Institute of Medical Sciences (AIIMS) technique' developed by the authors, in particular, is ingenious and perhaps seeks to function quite like a patent foramen ovale in early life where the septum primum serves as a valve that closes the defect in the atrial septum.

In order to arrive at any meaningful conclusions on the utility of unidirectional valved patches, it is vitally necessary to test the concept through systematically collected long-term follow-up data. A uniform recruitment process with clearly defined criteria for inclusion is necessary. Follow-up needs to be long because late attrition is common in CHD with PVOD. Survival curves in most published studies on outcomes after closure of shunts in the presence of PVOD appear to diverge after 4-5 years. ${ }^{5} 6$ It is important to recognise that available data clearly suggest that patients with CHD with corrected defects and residual pulmonary arterial hypertension (PAH) often have significantly worse long-term survival when compared with those with uncorrected defects. Therefore, follow-up for several years
(5-10 years or more) is of vital importance. Information on all domains needs to be meticulously recorded through a standardised protocol. This should include functional capacity, quality of life, patency and flow direction across fenestration, right ventricular function, pulmonary artery pressure and pulmonary vascular resistance (this will require a diagnostic catheterisation at specified intervals).

The results presented in this review essentially suggest that the unidirectional valve patch offers the prospect of surgical closure of left to right shunts in the presence of significant elevation in pulmonary vascular resistance. However, there is a paucity of good quality follow-up data in the studies listed in this review. The patient numbers are small in each of the studies; follow-up is incomplete and not standardised. Information on quality of life and functional capacity has not been systematically collected. Inclusion criteria are not clearly stated and not uniform. The studies with haemodynamic data have a substantial loss to follow-up ( 30\%) and are of insufficient duration. Because of shortcomings in the quality of follow-up data the utility of the UVP remains unproven.

At present the following fundamental questions still remain unanswered for patients with CHD and $\mathrm{PAH}$ :

1. Who should be operated and who should be left alone?

2. Who among those operated should receive a fenestrated-valved patch or an UVP?

3. What is the intermediate and longterm follow-up of these patients?

4. Should pulmonary vasodilators be administered in all these patients on follow-up? If so, what agent(s) and for how long?

If we seek to make better informed decisions on common left to right shunts with elevations in pulmonary vascular resistance and 'borderline' operability, we should consider a standardised protocol for diagnostic evaluation, management and follow-up that can be adopted by multiple participating institutions from low-income and middle-income countries in a prospective registry. Data can be collected at specified intervals in a standardised fashion over several years, and will be of immense value in deciding on indications for closure of the shunt in this challenging group of patients. A large database will allow for a wide spectrum of clinical possibilities to be investigated. Given the large numbers that are seen in centres in low-income and middle-income countries, it should be possible to initiate 


\section{Editorial}

this effort. ${ }^{7}$ Indeed, this may be the only way we can seek to effectively answer the questions on management of $\mathrm{CHD}$ with PVOD that continue to remain unanswered for more than five decades since the advent of congenital heart surgery.

Competing interests None declared.

Provenance and peer review Commissioned; internally peer reviewed.

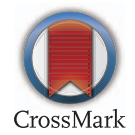

To cite Kumar RK. Heart Asia 2015;7:38-39. doi:10.1136/heartasia-2015-010680

\section{(P) Linked}

http://dx.doi.org/10.1136/heartasia-2015-010645

Heart Asia 2015;7:38-39.

doi:10.1136/heartasia-2015-010680

\section{REFERENCES}

1 Kumar RK, Shrivastava S. Pediatric heart care in India. Heart 2008;94:984-90.

2 Viswanathan S, Kumar RK. Assessment of operability in congenital cardiac shunts with increased pulmonary vascular resistance. Catheter Cardiovasc Interv 2008;71:665-70.

3 Galal AN, Barreto AC, Heath A, et al. Minima Requirements for Assigning PAH-CHD Patients to
Surgical Treatment According to the Opinion of the Pediatric Cardiologist and the Pediatrician. Pulm Circ 2014;4:330-41.

4 Talwar, Keshri VK, Choudhary SK, et al. Surgical strategies for patients with congenital heart disease and severe pulmonary hypertension in the developing world. Heart Asia 2015;7. doi:10.1136/heartasia2015-010645

5 Bonello B, Renard S, Mancini J, et al. Life span of patients with Eisenmenger syndrome is not superior to that of patients with other causes of pulmonary hypertension. Cardiovasc Diagn Ther 2014;4:341-9.

6 Haworth SG, Hislop AA. Treatment and survival in children with pulmonary arterial hypertension: the UK Pulmonary Hypertension Service for Children 2001-2006. Heart 2009;95:312-17.

7 Kumar RK. Distilling wisdom from our collective experience. Ann Pediatr Cardiol 2014;7:1-4. 